\title{
Construction and Characterization of an Acapsular Mutant of Pasteurella multocida Strain P-1059 (A:3)
}

Yu-Feng Zhang, Nazierbieke Wulumuhan, Feng-Juan Gong and Borrathybay Entomack*

College of Biology and Environmental Sciences, Jishou University, Jishou, Hunan, China

\begin{abstract}
To further investigate the role of capsule involved in virulence of Pasteurella multocida P-1059 (A:3), a hexB deleted mutant was constructed by homologous recombination. The DNA replacement was confirmed by PCR Reverse transcription (RT)-PCR and DNA sequencing. Experiments were conducted to compare the differences of biological characteristics such as capsular structure, capsular polysaccharide content, virulence and serum resistance between the hexB deleted mutant of $\Delta$ hexB and wild-type strain P-1059, as well as the complemented strain P-1059C. And the ability of the acapsular mutant $\Delta$ hexB to induced protection against wild-type challenge in chickens. Electron microscopy examination of the $\Delta$ hexB showed the absence of capsular material compared to the $\mathrm{P}-1059$ and $\mathrm{P}-1059 \mathrm{C}$. The $\Delta$ hexB was sensitive to the bactericidal action of chicken serum, whereas the P-1059 and P-1059C were both resistant. The $\Delta$ hexB was highly attenuated in chickens by intravenously injection, and intramuscular administration of $\Delta$ hexB to chickens stimulated significant protection against P-1059 and the homologous strain $\mathrm{X}-73(\mathrm{~A}: 1)$. These results demonstrated that the capsule is a major virulence factor of Pasteurella multocida serotype $A: 3$ strains.
\end{abstract}

Keywords: Pasteurella multocida; Homologous recombination; Knockout; Capsule; Virulence; Vaccine

\section{Introduction}

Pasteurella multocida causes fowl cholera in turkeys and chickens, and many avian species, and hemorrhagic septicemia in cattle and buffalos, and atrophic rhinitis in swine. The capsule of $P$. multocida type $\mathrm{A}$ is most often associated with avian cholera, and functions as a virulence factor, and it is composed largely of hyaluronic acid $[1,2]$. Strains belonging to capsular types B, D and F of $P$. multocida have also been isolated from diseased birds, but with low incidence as compared to capsular type A $[3,4]$.

The capsulated strains were more virulent than the noncapsulated strains of the virulent $P$. multocida, and the noncapsulated strains of virulent isolates are able to infect, but not to cause mortality [5,6]. A spontaneous noncapsulated mutant P-1059B obtained from 35 serial passages of $P$. multocida strain P-1059, demonstrated that the loss of ability to produce capsular materials resulted in a marked loss of virulence [7]. A capsulated strain P-1059 was shown to resist the action of complement compared to a noncapsulated strain P-1059-1A [8]. The capsular hyaluronic acid also mediated adhesion of $P$. multocida type A strains to turkey air sac macrophages [9]. The capsulated strain of $P$. multocida treated with hyaluronidase became complementsensitive and were more readily phagocytosed in comparison with untreated capsulated strain [10]. These studies have suggested that the capsular hyaluronic acid is a key virulence factor of $P$. multocida type A strains. However, because these strains were not genetically defined, it is not possible to ascribe definitively their phenotypes to the lack of capsule. Thus the entire capsule biosynthetic locus has been cloned and sequenced from a serotype A:1 strain X-73 of $P$. multocida [11], and sequence analysis showed that the locus containing three functional regions. Subsequently, constructed a defined acapsular mutant of the strain X-73 by disrupting the hexA gene through the insertion of a tetracycline resistance cassette, demonstrated that the capsule of the organism is an essential virulence factor in both mice and chickens [12]. In this study, we constructed an acapsular mutant of P. multocida P-1059 by homologous recombination, and pathogenicity in chickens and protective ability of the mutant strain were evaluated.

\section{Materials and Methods}

\section{Bacterial strains and plasmids}

Bacterial strains and plasmids used in this study are listed in table 1.

\begin{tabular}{|c|c|c|}
\hline Strain or plasmid & Relevant features & References \\
\hline \multicolumn{3}{|l|}{ Strains } \\
\hline E. coli DH5a & $\begin{array}{c}F^{-} \text {endA1 hsdR17 }\left(r_{k}^{-} m_{k}^{+}\right) \text {thi-1 } \lambda \text { recA1 } \\
\text { Ф80dlacZ } \Delta M 15\end{array}$ & TaKaRa \\
\hline \multicolumn{3}{|l|}{ P. multocida } \\
\hline $\mathrm{X}-73$ & Serotype A:1 wild-type strain & ATCC \\
\hline P-1059 & Serotype A:3 wild-type strain & ATCC15742 \\
\hline$\Delta$ hexB & The hexB deletion mutant of $\mathrm{P}-1059$ & This study \\
\hline P-1059C & $\begin{array}{l}\Delta \text { hexB complemented with plasmid } \\
\text { pPBA1101-hexB }\end{array}$ & This study \\
\hline \multicolumn{3}{|l|}{ Plasmids } \\
\hline pMD18-T & Cloning vector; Ampr & TaKaRA \\
\hline pBR322 & Cloning vector $\mathrm{Amp}^{r}$, Tetr & TaKaRA \\
\hline pWSK29 & $\begin{array}{l}\text { Low-copy-number E. coli cloning } \\
\text { vector, Ampr }\end{array}$ & {$[24]$} \\
\hline pPBA1101 & E. coli-P. multocida shuttle vector, $\mathrm{Kan}^{r}$ & [23] \\
\hline pMD18-hexABC & $\begin{array}{c}\text { The hexB gene and its flanking region } \\
\text { was cloned into pMD18-T, Amp }\end{array}$ & This study \\
\hline pWSK29 $2 h e x B$ & $\begin{array}{l}\text { pWSK29 containing Tetrgene, and } \\
\text { flanking region of the hexB gene; } \\
\text { Ampr } \text { Tet }^{r}\end{array}$ & This study \\
\hline pPBA1101-hexB & $\begin{array}{c}\text { pPBA1101 containing the hexB gene, } \\
\text { Kanr }^{r}\end{array}$ & This study \\
\hline
\end{tabular}

Table 1: Bacterial strains and plasmids used in this study.

*Corresponding author: Borrathybay Entomack, College of Biology and Environmental Sciences, Jishou University, Jishou, Hunan, 416000, China, E-mail: etmkb@jsu.edu.cn

Received April 04, 2013; Accepted April 26, 2013; Published April 28, 2013 Citation: Zhang YF, Wulumuhan N, Gong FJ, Entomack B (2013) Construction and Characterization of an Acapsular Mutant of Pasteurella multocida Strain P-1059 (A:3). J Vaccines Vaccin 4: 184. doi:10.4172/2157-7560.1000184

Copyright: ( 2013 Zhang YF, et al. This is an open-access article distributed under the terms of the Creative Commons Attribution License, which permits unrestricted use, distribution, and reproduction in any medium, provided the original author and source are credited. 
Escherichia coli DH5a containing recombinant plasmid was cultured in LB-broth (Difco, USA) or on LB agar plate at $37^{\circ} \mathrm{C}$. P. multocida strains were cultured in tryptose broth (Difco, USA) or on Dextrose starch agar (Difco, USA) at $37^{\circ} \mathrm{C}$. When required, broth or agar was supplemented with ampicillin $(100 \mu \mathrm{g} / \mathrm{ml})$, kanamycin $(50 \mu \mathrm{g} / \mathrm{ml})$ or tetracycline $(10$ $\mu \mathrm{g} / \mathrm{ml}$ for E. coli and $5 \mu \mathrm{g} / \mathrm{ml}$ for P. multocida).

\section{Cloning and sequencing of hexB gene and its flanking sequence}

For the PCR amplification, two specific primers P-1 ( $5^{\prime}$-ATGATCGAAACAAAAATAC-3') and P-2 (5'-CCCTATTCTTATTTACATG-3') were designed according to the published hexB gene and its flanking sequence of $P$. multocida X-73 [11]. The expected size of the resulting amplicon was 2909 bp in length. Genomic DNA of P. multocida P-1059 was isolated using the bacterial genomic DNA extraction kit (TaKaRa, China) according to the manufacturer's instructions. The hexB gene and its flanking sequence was amplified from the genomic DNA of P. multocida P-1059 by PCR using the primers P-1 and P-2. The PCR product was electrophoresed on a $1.0 \%$ agarose gel, and purified using an agarose gel DNA fragment recovery kit (TaKaRa, China) according to the manufacturer's instructions. The PCR product was cloned into a pMD18-T vector to generate pMD18-hexABC. The construct was transformed into chemically competent $E$. coli DH5a. The recombinants were selected onto LB plates containing $100 \mu \mathrm{g} / \mathrm{ml}$ ampicillin, $0.5 \mathrm{mM}$ IPTG and $80 \mu \mathrm{g} / \mathrm{ml} \mathrm{X-Gal}$. The recombinant plasmid DNA was isolated using a plasmid purification kit (TaKaRa), and the sequence of this insert was determined.

\section{Construction of targeting vector pWSK29}

The PCR primers were designed according to the sequenced hexB gene and its flanking sequence of P. multocida P-1059 (GenBank accession number JX679409) by using the primer premier 5.0 software program. The nucleotide sequences of these primers are described in table 2. The tetracycline resistance $\left(\mathrm{Tet}^{\mathrm{r}}\right)$ gene was amplified from pBR322 plasmid by PCR using primers Tet-F and Tet-R (Table 2), and digested with Sma I/Xho I and inserted into the same restriction sites of the plasmid pWSK29 to generate pWSK29-Tetr. DNA fragments of 991 bp (with PCR primers P991F and P991R) and 976 bp (with PCR primers P976F and P976R) upstream and downstream of the hexB gene were amplified by PCR and digested with Xba I /Sma I and Xho I/Kpn I, respectively. The two DNA fragments were inserted into the multiple cloning sites of the plasmid pWSK29-Tet to generate the targeting vector pWSK29 $\Delta$ hexB.

\section{Construction of $P$. multocida hexB deletion mutant}

Competent cells were prepared according to the previously

\begin{tabular}{|c|c|c|c|}
\hline Name & Sequence & $\begin{array}{l}\text { Restriction } \\
\text { site }\end{array}$ & $\begin{array}{c}\text { PCR } \\
\text { product }\end{array}$ \\
\hline P991F & 5'-CGCTCTAGATTTCTCAATCGAGTTTTTGTTGT-3' & Xba I & $991 \mathrm{bp}$ \\
\hline P991R & 5'-CGCCCCGGGTAATTTTTATGGCTCTCGAATGC-3' & Sma I & \\
\hline P976F & 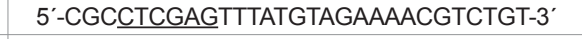 & Xhol & 976 bp \\
\hline P976R & 5'-GCGGGTACCCCCTATTCTTATTTACATG-3' & Kpn I & \\
\hline Tet-F & 5'-GCGCCCGGGATGAAATCTAACAATGCGCTCATCG-3' & Sma I & $1191 \mathrm{bp}$ \\
\hline Tet-R & 5'-CGCCTCGAGTCAGGTCGAGGTGGCCCG-3' & Xhol & \\
\hline P687F & 5'-ATAGAGTGATTGGTGCTCTC-3' & & $687 \mathrm{bp}$ \\
\hline P687R & 5'-TTAAGTCACATAAAATGAGG-3' & & \\
\hline HexB-F & 5'-CGCGAGCTCATGTTATACGATGACC-3' & Sac 1 & 798 bp \\
\hline HexB-R & 5'-CGCTCTAGATCATCGAGGTTCTATC-3' & Xbal & \\
\hline
\end{tabular}

Table 2: Oligonucleotide primers used in this study. described method [13] with slight modification. A $100 \mathrm{ml}$ culture of P. multocida P-1059 cells was grown in TB medium to midlog phase and treated with 100 units/ml of hyaluronidase (Sigma, USA) for $1 \mathrm{~h}$ to remove capsule. The bacterial cells were harvested by centrifugation at $4000 \times \mathrm{g}$ for $15 \mathrm{~min}$, and washed with sterile water for three times at $4^{\circ} \mathrm{C}$. The bacterial pellet was suspended in $1 \mathrm{ml}$ ice-water and placed on ice. The competent cells $(40 \mu \mathrm{l})$ were mixed with $10 \mu \mathrm{g}$ of the targeting vector pWSK29 2 hexB in $0.1 \mathrm{~cm}$ electroporation cuvette (Bio-Rad). Immediately after adding DNA, the cells were electroporated (Gene Pulser, Bio-Rad) at $2.5 \mathrm{kV}, 25 \mu \mathrm{F}, 600 \Omega$ with resultant time constants ranging 11 to $15 \mathrm{~ms} .1 \mathrm{ml} \mathrm{TB}$ medium was added to the electroporated cells and the cells were then recovered at $37^{\circ} \mathrm{C}$ for $3 \mathrm{~h}$, and plated onto DSA plates containing $5 \mu \mathrm{g} / \mathrm{ml}$ of tetracycline. Colonies were visible after $48 \mathrm{~h}$ incubation at $37^{\circ} \mathrm{C}$, and the tetracycline resistance colonies were screened by colony PCR for the presence or absence of the hexB gene in the genomic DNA of putative mutant strains using primers P687F and P687R (Table 2).

\section{PCR analysis of mutant locus}

After replacing the hexB gene in P. multocida P-1059 by the Tet gene in targeting vector pWSK29 $\Delta$ hexB, the hexB-deleted strain was obtained. The mutant locus in genomic DNA of this mutant was confirmed by PCR using primer pairs P687F and P687R, P991F and P976R, respectively. Simultaneously, the PCR products were cloned into the pMD18-T vector using E. coli DH5a. The sequence of the inserts was determined to confirm mutant locus.

\section{Complementation of hexB deletion mutant}

The entire hexB gene was amplified from genomic DNA of $P$. multocida P1-59 by PCR using primers HexB-F and HexB-R (Table 2). The PCR product was digested with $\mathrm{Sac} \mathrm{I} / \mathrm{X} b a \mathrm{I}$ and cloned into the same restriction sites of E. coli-P. multocida shuttle vector pPBA1101, generating pPBA1101-hexB, which was introduced into the hexB deletion mutant by electroporation to generate complemented strain P-1059C.

\section{RT-PCR analysis of hexB deletion mutant}

The P. multocida strains were grown to an optical density of 0.5 at $600 \mathrm{~nm}$, and the total RNA was isolated using the RNAprep pure Cell/Bacteria kit (Tiangen, China) according to the manufacturer's instructions. The RNA was treated with DNase to eliminate contaminating DNA, and the cDNA was synthesized using the random octamers provided in the Quantscript first-strand synthesis kit for RTPCR (Tiangen, China) according to the manufacturer's instructions. The primers used for RT-PCR analysis were P687F and P687R (Table 2). PCRs on the RT product and no RT control were performed with $E x$ Taq polymerase (TaKaRa, China) using standard procedures.

\section{Observation of capsular structure of $P$. multocida strains}

Bacterial cells were prepared for transmission electron microscopy as described previously [14]. Briefly, P. multocida strains were inoculated into fresh TB medium and incubated at $37^{\circ} \mathrm{C}$ for $6 \mathrm{~h}$. A 250 $\mu \mathrm{l}$ aliquot of the broth culture was spread on DSA plates and incubated at $37^{\circ} \mathrm{C}$ for $18 \mathrm{~h}$. The bacteria grown on the DNA plate were suspended in $0.1 \mathrm{M}$ cacodylate buffer containing 5\% glutaraldehyde and $0.15 \%$ ruthenium red for fixation and staining, respectively. The bacterial suspension was incubated at room temperature for $2 \mathrm{~h}$, and the bacteria were collected by centrifugation at $480 \times \mathrm{g}$ for $10 \mathrm{~min}$. The bacterial cells were suspended in $0.05 \mathrm{M}$ cacodylate buffer and allowed to react with a $1.0 \mathrm{mg} / \mathrm{ml}$ polycationic ferritin (Sigma) at room temperature for 
$30 \mathrm{~min}$. The reaction was stopped by 10 -fold dilution with cacodylate buffer, and the bacteria were washed three times in cacodylate buffer by centrifugation. The bacteria were then immobilized in $2 \%$ Noble agar, washed three times in cacodylate buffer, and postfixed with $2 \%$ osmium tetraoxide for $1 \mathrm{~h}$. The specimens were dehydrated in graded ethanols, and embedded in an epoxy resin mixture. Thin sections of the embedded specimen were stained with uranyl acetate and lead citrate, and then observed by electron microcopy at an acceleration voltage of $75 \mathrm{~kW}$ at calibrated magnification.

\section{Determination of hyaluronic acid capsule production}

Crude capsular polysaccharides were prepared according to the previously described method [15] with slight modification. Overnight cultures of $P$. multocida strains P-1059, $\Delta$ hexB and its complemented strain P-1059C grown in TB medium were diluted in $20 \mathrm{ml}$ of fresh TB medium to an optical density of 0.1 at $600 \mathrm{~nm}$ and incubated at $37^{\circ} \mathrm{C}$ with aeration until mid-exponential phase $\left(O D_{600}\right.$ of $\left.\sim 0.5\right)$. Cells were harvested from $5 \mathrm{ml}$ of bacterial culture by centrifugation at 7,600 $\times \mathrm{g}$ for $15 \mathrm{~min}$, washed once with sterile phosphate-buffered saline (PBS; pH7.4), resuspended in $1 \mathrm{ml}$ of PBS and incubated at $42^{\circ} \mathrm{C}$ for $1 \mathrm{~h}$ to extract the capsular polysaccharides. Viable counts were determined before and after the incubation at $42^{\circ} \mathrm{C}$, after which the cells were harvested by centrifugation at $7,600 \times \mathrm{g}$ for $15 \mathrm{~min}$ and supernatant containing the capsular polysaccharide was transferred to a new tube. The hyaluronic acid content in the capsular extract was determined according to the previously described method [16].

\section{Virulence of $P$. multocida strains for chickens}

A total of 75 specific-pathogen-free chickens, Line 22 white Leghorn chickens (Verial Vital Laboratory Animal Technology Co. Ltd., China), approximately 56-day-old were used for testing the virulence of $P$. multocida strains P-1059, $\Delta$ hexB and P-1059C. These strains were grown in TB medium to an optical density of 0.5 at 600 $\mathrm{nm}$, and the cultures were diluted into TB medium to obtain cultures of the desired concentrations. Exact bacterial numbers in the dilutions were determined by colony plate counts of serial dilutions. Each strain was inoculated in five groups of five birds each. The chickens were intravenously inoculated with $0.3 \mathrm{ml}$ of serial dilution bacterial cultures. Five birds served as untreated control. The chickens were kept in plastic isolators and observed for clinical signs for one week after inoculation.

\section{Serum sensitivity assays}

The sensitivity of P. multocida strains and E. coli DH5a to chicken serum was determined according to the previously described method [17] with slight modification. Briefly, whole blood was obtained from a SPF chicken, and the serum was separated by centrifugation at $800 \times \mathrm{g}$ for 15 min. P. multocida strains grown on DSA plates or E. coli DH5a grown on $\mathrm{LB}$ plates at $37^{\circ} \mathrm{C}$ for $18 \mathrm{~h}$ were suspended in $0.1 \mathrm{M}$ phosphatebuffered saline (PBS, pH 7.4), and the bacterial suspensions were adjusted to the concentration of approximately $1 \times 10^{6} \mathrm{CFU} / \mathrm{ml}$. Cells were harvested from $1 \mathrm{ml}$ of the bacterial suspension by centrifugation at $800 \times \mathrm{g}$ for $15 \mathrm{~min}$, and the bacterial cells were suspended in an equal volume of $90 \%$ serum at $37^{\circ} \mathrm{C}$ for $3 \mathrm{~h}$. Complement activity was inactived in control samples by heating at $56^{\circ} \mathrm{C}$ for $30 \mathrm{~min}$. The sensitized bacterial samples were diluted 10 -fold and plated onto DSA plates or LB plates, following which the number of viable cells was determined by direct colony counts on DSA agar. All assays were conducted in triplicate for $P$. multocida strains and E. coli DH5a. Serum sensitivity between the $P$. multocida P-1059 $\Delta$ hexB and P-1059C were compared for statistical significance using the Student's $t$-test.

\section{Protection assay}

Line 22 white Leghorn chickens (Verial Vital Laboratory Animal Technology Co. Ltd., China), approximately 56-day-old were used. Chickens were divided into 4 groups based on the strains for challengeexposure (Table 6). Groups 1 and 3 were vaccinated with a live vaccine as an experiment groups while groups 2 and 4 were vaccinated with a sterile BHI broth as negative groups. Chickens of groups 1 and 3 were vaccinated with a single dose of a live vaccine with the concentration of $3.6 \times 10^{8} \mathrm{CFU} / \mathrm{ml}$. Chickens were challenge-exposed at two weeks post vaccination. Chickens of groups 1 and 2 were challenge-exposed with $4.5 \times 10^{3} \mathrm{CFU} / \mathrm{ml}$ of the parent strain P-1059 to determine the homologous protection, while groups 3 and 4 were challenge-exposed with $3.7 \times 10^{3} \mathrm{CFU} / \mathrm{ml}$ of strain to determine the heterologous protection. The birds were observed for their mortality rates and clinical signs for ten days.

\section{Results}

\section{Cloning and sequencing of the hexB gene and its flanking sequence}

As shown in figure 1, a $2.9 \mathrm{~kb}$ fragment was successfully amplified from the genomic DNA of P-1059 by PCR. The PCR product was cloned into the pMD18-T vector and the nucleotide sequence of the inserts was determined. The DNA fragment was 2909 bp in length comprising three ORFs representing the three capsule transport protein genes hexCBA. The hexC contains 1137 nucleotides and terminates at a TAA stop codon, encoding a putative protein of 378 amino acids. The third base of the stop codon at the 3 -end of hexC is the first base of the ATG at the start of hexB, 798 nucleotides in length and coding for a putative protein of 265 amino acids. The hexB terminates with a TGA stop codon where it overlaps with hexA, the nucleotides ATGA forming part of the start codon. The hexA containing 660 nucleotides, encoding a putative protein of 220 amino acids and terminates at a TAA stop codon. The DNA homology of the hexABC genes between the P. multocida P-1059 and the previous reported P. multocida X-73 in GenBank was 99\%.

\section{Construction of a targeting vector pWSK29 2 hexB}

The targeting vector pWSK29 $\Delta$ hexB was designed to delete the hexB gene encoding capsular hyaluronic acid export protein $\mathrm{B}$ in $P$. multocida $\mathrm{P}-1059$ by homologous recombination. The sequence analysis of targeting vector pWSK29 $\Delta$ hexB confirmed the presence of $1191 \mathrm{bp}$ tetracycline resistant gene, the $991 \mathrm{bp}$ hexB gene upstream fragment and the 976 bp hexB gene downstream segment of the hexB gene (data not shown). This indicates that the targeting vector pWSK29 $\Delta$ hexB was successfully constructed.

\section{Construction of hexB deletion mutant}

The targeting vector pWSK29 4 hexB was transformed to $P$. multocida P-1059 by electroporation. By homologous recombination, $\mathrm{Tet}^{r}$ gene replaced hexB gene. Thus the hexB deletion mutant was generated. Several colonies exhibiting tetracycline resistance phenotype and growing on the DSA plate (containing $5 \mu \mathrm{g} / \mathrm{ml}$ tetracycline) were picked, and the putative mutant strains were screened by colony PCR As shown in figure 2, using the primers P687F and P687R, a 687 bp fragment was amplified from the genomic DNA of parent strain P-1059. In contrast, no product was amplified from the genomic DNA of two putative mutants. These results indicated that the hexB gene was deleted from the genomic DNA of these strains. The hexB deletion strain was designated $\Delta h e x B$. 


\section{PCR analysis of mutant locus}

The genomic DNAs from P. multocida P-1059 and $\Delta$ hexB were prepared, respectively. PCR primers P991F and P976R were used to amplify the expected $2.7-\mathrm{kb}$ fragment from the genomic DNA of parent strain P-1059. The PCR results showed that a $3.2 \mathrm{~kb}$ fragment was amplified from the genomic DNA of $\Delta h e x B$. In constructing the targeting vector $\mathrm{pWSK} 29 \Delta$ hexB, a 1191-bp tetracycline resistant gene was replaced with the 798 bp hexB gene, accounting for the 393-bp difference in these PCR products (Figure 3 ). The sequences of the PCR products were cloned and confirmed by DNA sequencing.

\section{RT-PCR analysis of hexB deletion mutant}

The expression of the hexB was analyzed by RT-PCR of total RNA, using primers P687F and P687R. RT-PCR analysis showed that the expected 687-bp product was present in the parent strain P-1059 but was absent in the mutant $\Delta$ hexB (Figure 4). These results confirmed the hexB gene was successfully deleted by homologous recombination. Complementation of the $\Delta$ hexB mutant with plasmid pPBA1101-hexB restored the hexB transcript to $\Delta$ hexB (Figure 4).

\section{Observation of capsular structure of $P$. multocida strains}

P. multocida strains were cultured on the DSA plates at $37^{\circ} \mathrm{C}$ for 18 h. Parent strain P-1059 produced mucoid colonies, consistent with the presence of a capsule, while both the mutant $\Delta$ hexB and the complemented strain P-1059C produced nonmucoid and small colonies (data not shown). As shown in figure 5, the capsule of mutant $\Delta h e x B$ was thinner than that of the parental strain P-1059 according to electron microscopy. On the other hand, the complemented strain P-1059C had a thin and irregular capsule on bacterial cell surface.

\section{Hyaluronic acid capsule production}

The production of extracellular polysaccharide was determined by direct chemical assay for hyaluronic acid (Table 3). The viability of the cells was determined after the capsule extraction procedure and ranged from $10 \%$ to $30 \%$ of that prior to extraction. Mutant strain $\Delta$ hexB produced significantly less hyaluronic acid than did the parent strain P-1059 and complemented strain P-1059C $(P<0.01)$. No significant difference between the hyaluronic acid produced by strains P-1059 and $\mathrm{P}-1059 \mathrm{C}(P>0.05)$. These results suggested that the complemented strain P-1059C had restored the ability to transport extracellular hyaluronic acid.

\section{Virulence of $P$. multocida strains in chickens}

The results of virulence test of $P$. multocida strains are shown in table 4. The parent strain P-1059 killed all chickens by intravenous injection at a dose of $10^{1}$ to $10^{5} \mathrm{CFU}$. In contrast, no deaths were recorded for

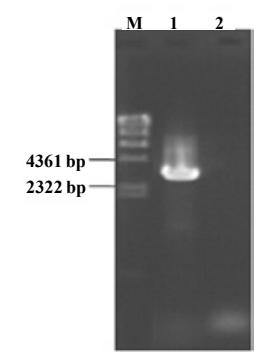

Figure 1: $P C R$ amplification of hexB gene and its flanking region from $P$. multocida $\mathrm{P}-1059$ using primers $\mathrm{P}-1$ and $\mathrm{P}-2$. lane $\mathrm{M}$ : $\lambda$-Hind digest DNA marker, lane 1: PCR product, lane 2: Negative control.

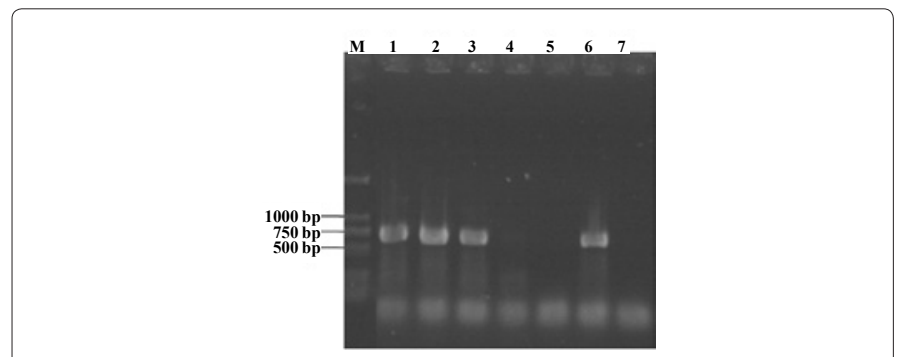

Figure 2: Identification of hexB mutants by colony PCR using the primers P687F and P687R. Lane M: DL2000 DNA marker, lane 2: PCR product of P-1059, lanes 2, 3 and 6: PCR-positive colonies, lanes 4 and 5: PCR-negative colonies, lane 8: Negative control.

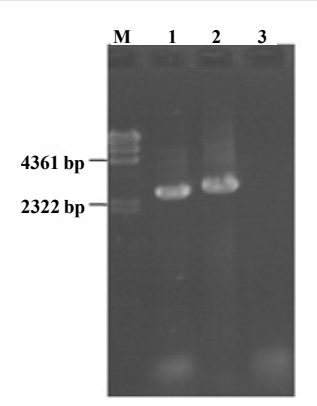

Figure 3: PCR verification of hexB deletion mutant of $\triangle$ hexB using the primers P991F and P976R. Lane M: $\lambda$-Hindlll digest DNA marker, lane 1: PCR product of parent strain P-1059, lane 2: PCR product of mutant $\Delta$ hexB, lane 3: negative control.

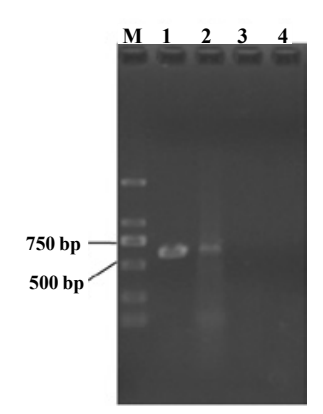

Figure 4: RT-PCR analysis of $P$. multocida strains using primers P687F and P687R. Lane M: DL2000 DNA marker, Lane 1: total RNA of P-1059, Lane 2: total RNA of P-1059C, Lane 3: total RNA of $\triangle$ hexB, Lane 4: Negative control.

chicken intravenous injection with $10^{4}$ to $10^{6} \mathrm{CFU}$ of the mutant $\Delta$ hexB. However, the mutant $\Delta$ hexB revealed $60 \%$ and $100 \%$ mortality at high dose of $10^{7} \mathrm{CFU}$ and $10^{8} \mathrm{CFU}$ by intravenous injection, and the $50 \%$ lethal dose $\left(\mathrm{LD}_{50}\right)$ was calculated to be approximately $5.14 \times 10^{7} \mathrm{CFU}$. On the other hand, the complemented strain P-1059C killed all chickens by intravenous injection at a dose of $10^{5}$ to $10^{7} \mathrm{CFU}$, whereas low doses of $10^{3}$ and $10^{4} \mathrm{CFU}$ of the complemented strain P-1059C resulted in $20 \%$ and $60 \%$ mortality, and the $\mathrm{LD}_{50}$ was calculated to be approximately $6.71 \times 10^{3} \mathrm{CFU}$. These results show that the hexB deletion mutant is highly attenuated for virulence. Bacterial isolation was positive in all the dead chickens but negative in the surviving chickens.

\section{Serum sensitivity assays}

P. multocida strains P-1059, $\Delta$ hexB and P-1059C were incubated in $90 \%$ chicken serum to determine their resistance to complementmediated killing. While a serum-sensitivity control of E. coli DH5a 


\begin{tabular}{|c|c|c|}
\hline P. multocida strain & Amounts of hyaluronic acid $\left(\mathbf{1 0}^{-8} \boldsymbol{\mu g} /\right.$ CFU) & Viability $^{b}$ \\
\hline P-1059 & $12.2 \pm 0.9$ & $0.2 \pm 0.1$ \\
\hline DhexB & $2.3 \pm 0.3$ & $0.4 \pm 0.3$ \\
\hline P-1059C & $11.4 \pm 0.6$ & $0.1 \pm 0.2$ \\
\hline
\end{tabular}

${ }^{a}$ The levels of hyaluronic acid produced by strains $\mathrm{P}-1059$ and $\mathrm{P}-1059 \mathrm{C}$ compared to that of $\triangle h e x B$ were significantly

different $(\mathrm{P}<0.01),{ }^{b}$ Viability is expressed as the ratio of bacterial number $(\mathrm{CFU} / \mathrm{ml})$ after treatment at $42^{\circ} \mathrm{C}$ to that before treatment at $42^{\circ} \mathrm{C}$.

Table 3: Extracellular hyaluronic acid produced by P. multocida strains.

\begin{tabular}{|c|c|c|c|}
\hline Strain & Injected dose (CFU) & Mortality $^{b}(\%)$ & Bacterial isolation \\
\hline \multirow[t]{5}{*}{ P-1059 } & $4.63 \times 10^{1}$ & $5 / 5(100)$ & + \\
\hline & $4.63 \times 10^{2}$ & $5 / 5(100)$ & + \\
\hline & $4.63 \times 10^{3}$ & $5 / 5(100)$ & + \\
\hline & $4.63 \times 10^{4}$ & $5 / 5(100)$ & + \\
\hline & $4.63 \times 10^{5}$ & $5 / 5(100)$ & + \\
\hline \multirow[t]{5}{*}{$\Delta h e x B$} & $6.46 \times 10^{4}$ & $0 / 5(0)$ & - \\
\hline & $6.46 \times 10^{5}$ & $0 / 5(0)$ & - \\
\hline & $6.46 \times 10^{6}$ & $0 / 5(0)$ & - \\
\hline & $6.46 \times 10^{7}$ & $3 / 5(60)$ & + \\
\hline & $6.46 \times 10^{8}$ & $5 / 5(100)$ & + \\
\hline \multirow[t]{5}{*}{ P-1059C } & $2.58 \times 10^{3}$ & $1 / 5(20)$ & + \\
\hline & $2.58 \times 10^{4}$ & $3 / 5(60)$ & + \\
\hline & $2.58 \times 10^{5}$ & $5 / 5(100)$ & + \\
\hline & $2.58 \times 106$ & $5 / 5(100)$ & + \\
\hline & $2.58 \times 10^{7}$ & $5 / 5(100)$ & + \\
\hline Control $^{c}$ & TB & $0 / 5(0)$ & - \\
\hline
\end{tabular}

${ }^{a} \mathrm{CFU}$ is colony forming units determined by viable count, ${ }^{b}$ number of dead birds per five birds,

${ }^{c}$ Nontreated control chickens.

Table 4: Virulence of $P$. multocida strains in chickens.

was also examined to confirm the presence of complement activity in the serum. As shown in table 5, the parent strain P-1059 was not killed in chicken serum, and the average number of CFU per milliliter increased from $2.8 \times 10^{6}$ to $2.6 \times 10^{8}$ over $3 \mathrm{~h}$ of treatment. A similar trend was observed for the complemented strain P-1059C, increasing from an initial average of $3.2 \times 10^{6}$ to $3.6 \times 10^{7} \mathrm{CFU} / \mathrm{ml}$ over $3 \mathrm{~h}$ in chicken serum. However, the mutant $\Delta$ hexB was killed in chicken serum, and the average number of CFU per milliliter decreased from $2.9 \times 10^{6}$ to $2.3 \times 10^{3}$ over $3 \mathrm{~h}$ of incubation. Heat inactivated serum permitted growth of P. multocida strains P-1059, $\Delta$ hexB, P-1059C and E. coli DH5a. No statistically significant differences were observed between the bacteria number of P-1059, $\Delta$ hexB and P-1059C, after incubation in heat inactivity chicken serum.

\section{Protection assay}

Vaccines and results are shown in table 6. Chickens in group 1 was challenge-exposed with the parent strain P-1059, and complete protection (100\% survivor) was obtained. Chickens in group 3 was challenge-exposed with heterologous wild-type strain of X-73 (A:1), highly protection ( $80 \%$ survivor) was obtained.

\section{Discussion}

The entire capsule locus of avian P. multocida X-73 (A:1) was cloned and sequenced, and the locus was divided into three regions, the region 1 of which contains four genes, hexD, hexC, hexB and hexA are predicted to encode proteins responsible for transport of the polysaccharide to the bacterial surface [11]. The sequence analysis demonstrated that the P. multocida hexABCD were highly homologous at both nucleotide and amino acid levels to Haemophilus influenza bexABCD [18], Actinobacillus pleuropneumoniae cpxABCD [19] and Neisseria meningitidis ctrABCD [20]. In the serotype A:1 strain X-73, inactivation of the capsule transport gene hexA resulted in a mutant strain that was highly attenuated in both mice and chickens, and was more sensitive to the bactericidal activity of chicken serum [12].

Our previous study reported a noncapsulated mutant P-1059B obtained from 35 serial passages of P. multocida strain P-1059, demonstrated that the loss of ability to produce capsular materials resulted in a marked loss of virulence [7]. However, this study used spontaneously arising noncapsulated mutant. Thus in this study, we have constructed a hexB deletion mutant in the serotype A:3 strain P-1059, designated $\Delta h e x B$, and the $\Delta h e x B$ was observed to be nonmucoid colony, and the cells of $\Delta$ hexB appeared acapsular by electron microscopy compared to the parent strain P-1059. An intact copy of hexB in the E. coli-P. multocida shuttle vector pPBA1101 was introduced into the mutant $\triangle$ hexB to complement the deleted hexB, with resultant strain designated P-1059C, and the P-1059C revealed a thick capsule material only on some cells. According to the hypothesis of previous report [12], the amount of extracellular capsule produced may not reflect its distribution on the surface of the cell. This result demonstrated that the hexB gene of $P$. multocida type A strain was responsible for transport of the polysaccharide to the bacterial surface.

Previous study reported the capsule as a virulence factor for chickens in an fowl cholera-causing $P$. multocida serotype A:1 strain by using a defined acapsular mutant [12]. In this study, the capsule was shown to be a virulence factor for $P$. multocida serotype A:3 strain in the chickens, by using the hexB deletion mutant. The acapsular mutant $\triangle$ hexB low virulence at a high dose as compared with the parent strain P-1059. When the intact hexB gene was restored in the complemented strain P-1059C, the ability to cause lethal infection was restored approximately to parent strain P-1059 levels in chickens. These results confirm previous work that capsule is major virulence factor in the pathogenesis of fowl cholera and show specifically that capsule is a critical virulence factor in the serotype A:3 strain.

\begin{tabular}{|c|c|c|}
\hline Strains & Serum heat treatment & Survival ratio \\
\hline E. coli DH5a & - & $0^{a}$ \\
\hline P. multocida & + & $8.6^{a}$ \\
\hline P-1059 & - & $165 \pm 57$ \\
\hline & + & $196 \pm 64$ \\
\hline ShexB & - & $0.24 \pm 0.07^{a}$ \\
\hline & + & $126 \pm 22^{a}$ \\
\hline & - & $97 \pm 19^{a}$ \\
\hline & + & $142 \pm 26^{a}$ \\
\hline
\end{tabular}

a Significantly different at $\mathrm{P}<0.01$ when compared to serum and heated serum treatment

Table 5: Resistance of $E$. coli DH5 and P. multocida strains to chicken serum.

\begin{tabular}{|c|c|c|c|}
\hline $\begin{array}{c}\text { Group of } \\
\text { chicken }\end{array}$ & $\begin{array}{c}\text { Challenge dose } \\
\text { (CFU) }\end{array}$ & $\begin{array}{c}\text { Challenge strain } \\
\text { (serotype) }\end{array}$ & $\begin{array}{c}\text { Survival after } \\
\text { challenge }\end{array}$ \\
\hline 1 & $4.5 \times 10^{3}$ & P-1059 (A:3) & $5 / 5$ \\
\hline 2 & BHI broth & P-1059 (A:3) & $0 / 5$ \\
\hline 3 & $3.7 \times 10^{3}$ & X-73 (A:1) & $4 / 5$ \\
\hline 4 & BHI broth & X-73 (A:1) & $0 / 5$ \\
\hline
\end{tabular}

Table 6: Protection confirmed in chickens by immunization of live $P$. multocida $\Delta$ hexB against challenge with $P$. multocida strains. 


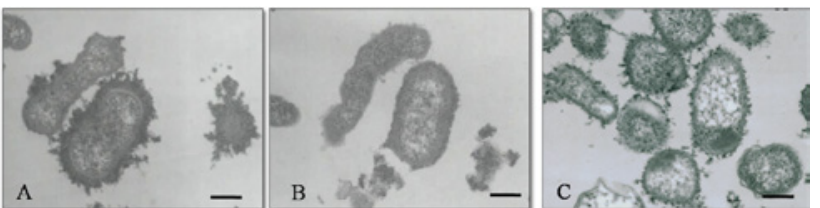

Figure 5: Electron micrographs of P. multocida strains P-1059 (A), P-1059C (B) and $\Delta$ hexB (C). Bars, $200 \mathrm{~nm}$

The result of serum resistance was consistent with a role for the serotype A capsule in survival in vivo. The parent strain P-1059 and complemented strain P-1059C were resistance to the bactericidal action of chicken serum, while acapsular mutant $\Delta$ hexB was highly sensitive. This agrees with previous results obtained by using spontaneously derived acapsular mutant and enzymatic removal of capsule [21] or using a defined acapsular mutant PBA930 [12]. These results demonstrated that the capsule of $P$. multocida serotype A:3 strains was responsible for protection against the bactericidal activity of complement, and the ability of $\Delta$ hexB to induce protection against both homologous and heterologous wild-type strains was similar to that of the acapsular mutant PBA930 [22].

In conclusion, we successfully constructed a genetically defined acapsular mutant of a serotype A:3 strain by homologous recombination. It was shown that deletion of the hexB gene resulted in the loss of surface-expressed capsular polysaccharide in this mutant. In chicken serum, the mutant $\triangle$ hexB was killed to a greater degree than the parent strain P-1059, indicating that the capsule of the P. multocida serotype A:3 strain is mediating resistance to serum bacteriolysis through the classical complement pathway. Moreover, the hexB deletion mutant is highly attenuated for virulence $[23,24]$.

\section{Acknowledgments}

The present study was supported by the National Natural Science Foundation of China (30972206). We are grateful to Professor Ben Adler at Monash University in Australia, for providing the plasmid pPBA1101 and the excellent technical assistance.

\section{References}

1. Rosner H, Grimmecke HD, Knirel YA, Shashkov AS (1992) Hyaluronic acid and a $(1 \rightarrow 4)-\beta$-D-xylan, extracellular polysaccharides of Pasteurella multocida (Carter type A) strain 880. Carbohydr Res 223: 329-333.

2. Pandit KK, Smith JE (1993) Capsular hyaluronic acid in Pasteurella multocida type $A$ and its counterparts in type D. Res Vet Sci 54: 20-24

3. Rhoades KR, Rimler RB (1987) Capsular groups of Pasteurella multocida isolated from avian hosts. Avian Dis 31: 895-898

4. Rhoades KR, Rimler RB, Bagley RA (1992) Fowl cholera epornitic: antigenic characterization and virulence of selected Pasteurella multocida isolates. Avian Dis 36: $84-87$

5. Tsuji M, Matsumoto M (1989) Pathogenesis of fowl cholera: influence of encapsulation on the fate of Pasteurella multocida after intravenous inoculation into turkeys. Avian Dis 33: 238-247.
6. Dubreuil JD, Gilbert L, Jacques M (1992) Cell surface characteristics and virulence in mice of Pasteurella multocida. Zentralbl Bakteriol 276: 366-373.

7. Borrathybay E, Sawada T, Kataoka Y, Okiyama E, Kawamoto E, et al. (2003) Capsule thickness and amounts of a $39 \mathrm{kDa}$ capsular protein of avian Pasteurella multocida type A strains correlate with their pathogenicity for chickens. Vet Microbiol 97: 215-227.

8. Snipes KP, Hirsh DC (1986) Association of complement sensitivity with virulence of Pasteurella multocida isolated from turkeys. Avian Dis 30: 500-504

9. Pruimboom IM, Rimler RB, Ackermann MR, Brogden KA (1996) Capsular hyaluronic acid-mediated adhesion of Pasteurella multocida to turkey air sac macrophages. Avian Dis 40: 887-893.

10. DeAngelis PL, Jing W, Drake RR, Achyuthan AM (1998) Identification and molecular cloning of a unique hyaluronan synthase from Pasteurella multocida. J Biol Chem 273: 8454-8458

11. Chung JY, Zhang Y, Adler B (1998) The capsule biosynthetic locus of PasteurellamultocidaA:1. FEMS Microbiol Lett 166: 289-296.

12. Chung JY, Wilkie I, Boyce JD, Townsend KM, Frost AJ, et al. (2001) Role of capsule in the pathogenesis of fowl cholera caused by Pasteurella multocida serogroup A. Infect Immun 69: 2487-2492.

13. Tatum FM, Yersin AG, Briggs RE (2005) Construction and virulence of Pasteurella multocida fhaB2 mutant in turkeys. Microb Pathog 39: 9-17.

14. Jacques M, Foiry B (1987) Electron microscopic visualization of capsular material of Pasteurella multocida type $A$ and $D$ labeled with polycationic ferritin J Bacteriol 169: 3470-3472.

15. Gentry MJ, Corstvet RE, Panciera RJ (1982) Extraction of capsular materia from Pasteurella haemolytica. Am J Vet Res 43: 2070-2073.

16. Moses AE, Wessels MR, Zalcman K, Albertí S, Natanson-Yaron S, et al. (1997) Relative contributions of hyaluronic acid capsule and $M$ protein to virulence in a mucoid strain of the group A Streptococcus. Infect Immun 65: 64-71.

17. McKerral LJ, Lo RY (2002) Construction and characterization of an acapsula mutant of Mannheimia haemolytica A1. Infect Immun 70: 2622-2629.

18. Kroll JS, Loynds B, Brophy LN, Moxon ER (1990) Thebex locus in encapsulated Haemophilus influenzae: a chromosomal region involved in capsule polysaccharide export. Mol Microbiol 4: 1853-1862.

19. Ward CK, Inzana TJ (1997) Identification and characterization of a DNA region involved in the export of capsular polysaccharide by Actinobacillus pleuropneumoniae serotype 5a. Infect Immun 65: 2491-2496.

20. Frosch M, Müller D, Bousset K, Müller A (1992) Conserved outer membrane protein of Neisseria meningitidis involved in capsule expression. Infect Immun 60: 798-803.

21. Jacques M, Kobisch M, Bélanger M, Dugal F (1993) Virulence of capsulated and noncapsulated isolates of Pasteurella multocida and their adherence to porcine respiratory tract cells and mucus. Infect Immun 61: 4785-4792.

22. Chung JY, Wilkie I, Boyce JD, Adler B (2005) Vaccination against fowl cholera with acapsular Pasteurella multocida A:1. Vaccine 23: 2751-2755.

23. Homchampa P, Strugnell RA, Adler B (1997) Cross protective immunity conferred by a marker-free aroA mutant of Pasteurella multocida. Vaccine 15 203-208.

24. Wang RF, Kushner SR (1991) Construction of versatile low-copy-number vectors for cloning, sequencing and gene expression in Escherichia coli. Gene 100: 195-199. 